\title{
Predictive Value of Renal Function and Glomerular Filtration Rates in the Intensive Care Unit
}

\section{Gutiérrez HB* and Navas JL}

Intensive and Emergency Medicine, University Clinical Surgical Hospital, Havana, Cuba

*Corresponding author: Hedgar Berty Gutiérrez, Intensive and Emergency Medicine, University Clinical Surgical Hospital, Havana, Cuba, Tel: 77802129; E-mail: hedgar@infomed.sld.cu

Received Date: June 21, 2018; Accepted Date: July 17, 2018; Published Date: July 24, 2018

Copyright: ( 2018 Gutiérrez HB, et al. This is an open-access article distributed under the terms of the Creative Commons Attribution License, which permits unrestricted use, distribution, and reproduction in any medium, provided the original author and source are credited.

\begin{abstract}
Introduction: Acute renal failure (ARF) is a common condition in the intensive care unit (ICU) and probably a consequence of critical illness that is associated with adverse clinical outcomes, high costs and high mortality.
\end{abstract}

Objective: To determine the predictive capacity of renal function and estimated glomerular filtration rates (eGFR) on mortality in patients admitted to the ICU.

Method: Two hundred ninety patients admitted to the ICU of the University Hospital "Dr. Miguel Enriquez" between January 1, 2015 and December 1, 2016. The average creatinine, eGFR, diuretic rhythm, need for hemodynamic support, mechanical ventilation, hemodialysis and diagnosis upon admission to the ICU were determined. The probability of survival between the groups with and without ARF was evaluated and the risk of dying of the patients was determined using the Cox regression model.

Results: Sixty and six patients died in the ICU (23\%). The value of serum creatinine was higher; as well as the eGFR and the diuretic rhythm $(p<0.0001)$ lower in the group of patients who died compared to the survivors. The survival capacity was higher in patients who did not present with ARF. The eGFR (CKD-EPI) was the only independent predictor of mortality (AHR 0.92 [0.85-0.99], $p=0.026)$.

Conclusions: The assessment of renal function by estimating eGFR (CKD-EPI) provides useful and very valuable information for the initial evaluation of the critical patient.

Keywords: Mortality; Intensive care unit; Acute kidney injury; Glomerular filtration rate; Creatinine

\section{Introduction}

ARF is a common problem in ICUs with a high level of associated mortality [1,2]. This is no more than a process of diverse etiology, characterized by the abrupt loss of renal function that has as a final result the elevation of nitrogenous substances accompanied or not by reduction or cessation of urinary flow [3]. From $5 \%$ to $15 \%$ of patients admitted to the ICU experience an acute deterioration of renal function, caused mainly by sepsis, hypovolemia, arterial hypotension, use of nephrotoxic drugs and contrast media [4]. It is currently estimated that mortality ranges between $40 \%$ and $60 \%$ [5]. In Europe, the annual incidence of ARF is between 200 and 500 cases per million inhabitants. In terms of intensive care patients, between $1 \%$ and $35 \%$ can develop the disease [6]. An analysis of data from the National Hospital Discharge Survey of the United States under the direction of the Center for Disease Control and Prevention showed an increase in ARF among hospitalized patients from 18 per 100,000 populations in 1980 to 365 per 100,000 populations in 2005 [7]. The same results were found in Cuba, where there is an increase in its presence in the intensive care field. In a study conducted at the hospital Dr. Luis Diaz Soto, Perez and editors found that, of 227 patients admitted to the ICU, 29/227 (13\%) developed ARF [8].
The early identification of patients at risk of ARF allows us to develop strategies with the aim of improving their prognosis through early diagnosis and treatment intervention. In addition, it enables the close monitoring of certain interventions such as the adjustment of drugs and the prevention of nephrotoxicity by various agents in highrisk patients. A poor estimate of renal function can lead to a patient not receiving treatment appropriate to their risk, which leads to an increase in mortality [9]. Creatinine has been classically the most used endogenous substance for the evaluation of renal function. However, the creatinine concentration is affected by several factors (muscle mass, sex, race, type of feeding), in addition to others related to the creatinine filtration itself, such as tubular secretion, production and extra renal excretion. Given the disadvantages of plasma creatinine as a marker of renal deterioration, there have been multiple proposed methods to understand renal function, within which the formulas for estimating glomerular filtration are the most commonly used in routine clinical practice because of their relative simplicity [9]. There are different formulas to estimate the glomerular filtration in a certain patient. All of them incorporate a series of variables that attempt to resolve the deficiencies of plasma creatinine as the only marker of renal function. However, its accuracy is not constant in all scenarios and there are still situations in which we do not have a reliable method to know the degree of real renal deterioration of the patient [9]. In fact, in critical patients these formulas have not been studied at the same level as in the chronic, it is even suggested that they do not have the same 
Page 2 of 5

validity due to possible variations of renal function in short periods of time [10].

In our country there are few studies that show the relationship between the patient in critical condition and acute kidney disease, although in the world literature there are works on this subject in which they associate the presence of acute kidney injury and its impact on mortality long term, that is, outside the scope of critical care. For this reason, the objective of this study is to determine the predictive capacity of renal function and glomerular filtration rates on mortality in patients admitted to the ICU.

\section{Methods}

\section{Design}

A cohort, observational, prospective study was carried out in the ICU of the University Clinical Surgical Hospital "Dr. Miguel Enriquez "located in the province of Havana between January 1, 2015 and December 1, 2016. The center has 430 beds for hospitalization and the ICU has eight beds. The study was approved with the Scientific Council and the Ethics Committee of the center. We included 392 patients admitted to the ICU of our hospital, who underwent the first 48 hours of admission, complementary studies that assess renal function. Acute renal failure (N17) was diagnosed according to the criteria of the KDIGO definition [11]. Patients with chronic renal failure (CRF) and those with incomplete medical records in the database were not evaluated. Subjects with an Acute Physiology and Chronic Health Evaluation II (Apache II) score lower than 10 and with a Grace (Global Registry of Acute Coronary Events) score of inhospital death of less than 140 were excluded. The sample consisted of patients who really presented a serious state of the pathology that caused their admission, thus reducing the risk of bias of selection in the study. We divided the population into two subgroups, initially according to the presence or absence of ARF at the time of admission, subsequently depending on the survival or death of the patients, and each of them was analyzed separately.

\section{Data collection}

From the ICU database, the following variables were taken at admission: age, sex, skin color, weight, body mass index (BMI), cause of admission to the ICU, use of mechanical ventilation, hemodynamic support, average creatinine, diuretic rhythm, need for hemodialysis, estimated glomerular filtration rate (eGFR) according to the Chronic Kidney Disease Epidemiology Collaboration (CKD-EPI) equation, Modification of Diet in Renal Disease (MDRD-4) and the CockcroftGault (CG), as well as the stay in ICU. The response variable was global mortality in ICU.

\section{Statistical analysis}

For all the variables, summary measures were used, so that the qualitative ones were expressed with absolute numbers and percentage, while the quantitative ones as mean with standard deviation (SD) or median with $25 \%-75 \%$ interquartile range (IQR) according to the distribution of normality of the population. The comparison between the study groups was performed using the Chi-square test $\left(\chi^{2}\right)$ or Fisher's exact test. For the quantitative variables, the Mann-Whitney $U$ test was used. The Kaplan-Meier method was used to estimate the survival probability of patients admitted to the ICU, and the Log-Rank statistic was determined to check whether there were differences between patients with and without ARF and the occurrence of the event. The Cox proportional hazard model with adjust hazard ratio (AHR) and 95\% confidence interval (CI) was used. Statistical tests were considered significant with a bilateral $\mathrm{p}$ value of less than 0.05 . The statistical analysis was performed using the IBM ${ }^{\circ}$ SPSS $^{\circ}$ program, version 23 (Chicago, IL, USA).

\section{Results}

During the study period, 392 patients were admitted to the ICU, of which 25 patients were not evaluated, 15 due to presenting CRF as a personal pathological history and 10 with incomplete clinical records in the database.

\begin{tabular}{|c|c|c|c|}
\hline \multirow{2}{*}{ Variable } & Without ARF & ARF & \multirow{2}{*}{$\mathbf{P}$} \\
\hline & $(\mathrm{N}=199)$ & $(\mathrm{N}=91)$ & \\
\hline Age, median (IQR*) & $62(52-72)$ & $65(52-74)$ & 0.101 \\
\hline Sex, n (\%) & \multicolumn{3}{|l|}{ - } \\
\hline Male & $123(61.8)$ & $55(60.4)$ & \multirow{2}{*}{0.194} \\
\hline Female & $76(38.2)$ & 36 (39.6) & \\
\hline Skin color, n (\%) & \multicolumn{3}{|l|}{-} \\
\hline Black & $90(45.2)$ & $44(48.4)$ & \multirow{2}{*}{0.206} \\
\hline White & $109(54.8)$ & $47(51.6)$ & \\
\hline Weight, median (IQR*) & $74(64-84)$ & $75(65-82)$ & 0.481 \\
\hline $\mathrm{BMI}$, average $\left(\mathrm{SD}^{* *}\right)$ & $24.9(4.08)$ & $25.7(4.35)$ & 0.838 \\
\hline Cause of income in ICU, $n(\%)$ & \multicolumn{3}{|l|}{-} \\
\hline Cardiovascular & $106(53.3)$ & $41(47.5)$ & $<0.0001$ \\
\hline Pulmonary & $20(10.1)$ & $6(6.6)$ & 0.968 \\
\hline Neurological & $3(1.5)$ & $1(1.1)$ & 0.224 \\
\hline Traumatic & $11(5.5)$ & $10(11)$ & $<0.0001$ \\
\hline Gastrointestinal & $29(14.6)$ & $12(13.2)$ & 0.788 \\
\hline Infectious & $4(2)$ & $10(11)$ & $<0.0001$ \\
\hline Gynecological & $1(0.5)$ & 0 & 0.772 \\
\hline Poisoning & $3(1.5)$ & $1(1.1)$ & 0.646 \\
\hline Other & $22(11,1)$ & $10(11)$ & 0.097 \\
\hline Hemodynamic support, n (\%) & $19(9,5)$ & $19(20,9)$ & $<0.0001$ \\
\hline Mechanical Ventilation, n (\%) & $25(12,6)$ & $23(25,3)$ & $<0.0001$ \\
\hline Stay in ICU, median (IQR*) & $3.0(2.0-5.0)$ & $4.0(2.0-6.0)$ & 0.097 \\
\hline
\end{tabular}

Table 1: General characteristics of the studied population, stratified according to the presence or not of ARF.

We excluded 37 patients with an Apache II score lower than 10 and 40 patients with Grace score of in-hospital cardiovascular death of less than 140 . We analyzed 290 subjects (74\%) and 66 subjects died in the ICU (23\%). The general characteristics of the studied population 
Page 3 of 5

stratified according to the presence or absence of ARF are shown in (Table 1).

Statistically significant differences were found in the diagnosis at admission of cardiovascular $(\mathrm{p}<0.0001)$, traumatic $(\mathrm{p}<0.0001)$ and infectious $(\mathrm{p}<0.0001)$ pathologies among patients with or without renal function impairment with respect to mortality. A powerful association was also observed in subjects requiring mechanical ventilation $(p<0.0001)$ and use of vasoactive amines $(p<0.0001)$ with the presence of acute renal injury and death in ICU.
Median mean creatinine and diuretic rate were $101.5 \mu \mathrm{mol} / \mathrm{L}$ (IQR $92-120 \mu \mathrm{mol} / \mathrm{L}$ ) and $1.7 \mathrm{~mL} / \mathrm{kg} / \mathrm{h}$ (IQR $1.2-2.1 \mathrm{ml} / \mathrm{kg} / \mathrm{h}$ ). The mean of the eGFR (CKD-EPI), the eGFR (MDRD-4) and the eGFR (CG) were $64 \mathrm{ml} / \mathrm{min} / 1.73 \mathrm{~m} 2\left(\mathrm{SD} 22.1 \mathrm{ml} / \mathrm{min} / 1.73 \mathrm{~m}^{2}\right), 65.1 \mathrm{ml} / \mathrm{min} / 1.73 \mathrm{~m}^{2}$ (SD $21.1 \mathrm{ml} / \mathrm{min} / 1.73 \mathrm{~m}^{2}$ ) and $64.1 \mathrm{ml} / \mathrm{min} / 1.73 \mathrm{~m}^{2}$ (SD $24.6 \mathrm{ml} / \mathrm{min} /$ $1.73 \mathrm{~m}^{2}$ ), respectively. With respect to dialysis treatment, only 16 patients $(5.5 \%)$ needed it. The distribution of the characteristics of renal function between patients who died and survivors are shown in (Table 2).

\begin{tabular}{|l|l|l|l|}
\hline \multirow{2}{*}{ Variable } & Dead & Live & P \\
\cline { 2 - 4 } & $\mathbf{( N = 6 6 )}$ & $\mathbf{( N = 2 2 4 )}$ & $<0.0001$ \\
\hline Average creatinine, median (IQR*) & $127.5(110-164.3)$ & $96(88,3-110)$ & $<0.0001$ \\
\hline eGFR (CKD-EPI), mean (SD**) & $47.2(18.7)$ & $69(20.6)$ & $<0.0001$ \\
\hline eGFR (MDRD-4), mean (SD**) & $49.5(18.6)$ & $69.7(19,5)$ & $<0.0001$ \\
\hline eGFR (CG), mean (SD**) & $47.5(19.8)$ & $69(23,7)$ & $<0.0001$ \\
\hline Diuretic rhythm, median (IQR*) & $1.0(0.8-1.6)$ & $1.8(1.5-2.1)$ & 0.129 \\
\hline Hemodialysis, $n(\%)$ & $6(9.1)$ & $10(4.5)$ & \\
\hline
\end{tabular}

CG: Cockcroft-Gault; CKD-EPI: Chronic Kidney Disease Epidemiology Collaboration; MDRD: Modification of Diet in Renal Disease; CI: Confidence Interval; AHR: Adjust Hazards Ratio; eGFR: Estimated Glomerular Filtration Rate.

Table 2: Characteristics of renal function.



Figure 1: Kaplan-Meier curve for the probability of survival in patients admitted to the ICU with and without a diagnosis of ARF. Note: ICU, Intensive Care Unit; ARF, Acute Renal Failure.

It was found that the distribution of serum creatinine was significantly higher in the group of patients who died compared to the group of survivors $(\mathrm{p}<0.0001)$.

On the other hand, the eGFR (CKD-EPI), the eGFR (MDRD-4), the eGFR (CG) and the diuretic rhythm were significantly lower among those who died in relation to those who did not die $(\mathrm{p}<0.0001)$.

The analysis of the Kaplan-Meier curve was performed (Figure 1).

\begin{tabular}{|l|l|l|l|}
\hline Variable & AHR & $\mathbf{9 5} \% \mathbf{C I}$ & $\mathbf{P}$ \\
\hline eGFR (CKD-EPI) & 0.92 & $0.85-0.99$ & 0.026 \\
\hline eGFR (MDRD-4) & 1.05 & $0.99-1.12$ & 0.108 \\
\hline eGFR (CG) & 0.99 & $0.97-1.02$ & 0.641 \\
\hline Mechanical Ventilation & 1.56 & $0.83-2.94$ & 0.167 \\
\hline Hemodynamic support & 1.25 & $0.64-2.44$ & 0.520 \\
\hline Hemodialysis & 0.63 & $0.23-1.70$ & 0.359 \\
\hline
\end{tabular}

CG: Cockcroft-Gault; CKD-EPI: Chronic Kidney Disease Epidemiology Collaboration; MDRD: Modification of Diet in Renal Disease; Cl: Confidence Interval; AHR: Adjust Hazards Ratio; eGFR: Estimated Glomerular Filtration Rate.

Table 3: Results of the Cox regression analysis.

Patients who did not have ARF had a higher probability of survival with a median stay of 16 days in the ICU, which revealed a statistically significant difference in relation to the group of patients who presented some degree of acute renal dysfunction with a Log Rank (Mantel-Cox) $(\mathrm{p}<0.0001)$.

In the Cox regression analysis (Table 3), of all the variables studied, only eGFR (CKD-EPI) was an independent predictor of mortality with a statistically significant relationship (AHR 0.92 [0.85-0.99], $\mathrm{p}=0.026$ ).

\section{Discussion}

It is now clearly established that there is an important relationship between kidney disease and cardiovascular disease. The prevalence of this disease among patients with cardiovascular disease is very high 
and has a prognostic character regardless of the presence of other cardiovascular risk factors, such as hypertension, diabetes mellitus, hypercholesterolemia or smoking. Within the broad spectrum of cardiovascular disease, ischemic heart disease and heart failure, are two of the most frequent groups of diseases in the ICU. The prognostic value of kidney disease has been amply demonstrated in both cardiovascular pathologies. In a multicenter prospective study with more than 1,000 hospitalized patients diagnosed with acute heart failure, the worsening of renal function during admission occurred in $27 \%$ of the patients and was independently associated with higher mortality; complications such as shock, myocardial infarction, stroke and development of atrial fibrillation; and longer duration of hospital stay [9]. In the ACTION (Acute Coronary Treatment and Intervention Outcome Network) registry, 57,700 patients with acute coronary syndrome were evaluated and it was shown that approximately 1 in 6 patients with acute myocardial infarction had acute kidney injury, which was associated with an increase in mortality [12]. The same results were found in the present study, where the diagnosis at the entrance of cardiovascular disease was statistically related to the presence of acute renal injury and mortality in the ICU.

There are a number of factors that relate ARF in patients with severe trauma, among which we have the reduction of perfusion with the consequent affectation to the permeability of the glomerular membrane, retrograde tubular filtration and tubular obstruction. In most cases the cause of this insufficiency is pre-renal, however, this also depends on the type of trauma that the patient presents, since in those with compression or crushing injuries the mechanism by which insufficiency is generated is intra-renal. The mechanisms of renal damage include critical reduction of renal perfusion, development of regional ischemia, direct tubulo-toxicity, decrease of intracellular ATP, action of endothelins, intracellular accumulation of calcium, deficiencies in cell growth factors and release of free radicals of oxygen [13]. In our study, severe trauma with ARF was significantly associated with mortality in the ICU.

Sepsis and its most severe form, septic shock, are the main causes of ARF in the ICU, corresponding to up to $50 \%$ of cases. Currently, it is proposed from the physiopathological point of view that the main mechanism of ARF in septic patients is ischemia or hypoperfusion, suggesting that the fall in renal blood flow and renal vasoconstriction would be the most characteristic events. There are also other factors than purely hemodynamic factors that participate in the genesis of ARF in sepsis. These include cellular apoptosis, glomerular and spinal microcirculatory disorders, cellular changes in responses to the pro inflammatory cascade characteristic of sepsis, oxidative stress and mitochondrial dysfunction [14]. Infections as a cause of admission, presented a powerful statistically significant relationship with respect to mortality and the presence of acute renal injury. Similar results were found by Angus et al. in 7 states of the United States, where 192,980 patients with a diagnosis of severe sepsis were examined [15], ARF occurred in $22 \%$ and mortality was associated in $38.2 \%$. In the cut-off study of the Occurring in Acutely Ill Patients (SOAP) in Europe, patients admitted in 198 Ucis were recruited. Of 3147 patients, $37 \%$ presented sepsis, ARF occurred in $51 \%$ of the cases and was associated with death in the ICU by $41 \%$ [15].

On the other hand, the use of vasoactive amines and the need for mechanical ventilation were also significantly associated with mortality in patients with acute kidney injury, this is related to the fact that all these variables suggest the presence of a more labile patient and a compromise parenchymal disease, which leads to the development of acute kidney damage. Severe acute kidney injury in critically ill patients is usually part of a triad with shock and respiratory failure requiring mechanical ventilation with positive pressure (PPV). The physiological effects of PPV and its effects on renal perfusion and function are well documented. Mechanical ventilation can induce renal dysfunction and acute tubular necrosis leading to ARF by three mechanisms: first, the effect of blood pressure on arterial blood, second, through the effect on systemic and renal arterial flow; finally, by means of the activation of a pulmonary inflammatory reaction, and the systemic release of mediators generated by biotrauma [5]. In a study conducted at the National Arzobispo Loayza Hospital, Lima, with patients who presented ARF during their stay in the ICU, (85\%) required the use of mechanical ventilation and (97.5\%) the use of vasoactive amines, both groups were associated with higher mortality [2].

Serum creatinine performed significantly higher and eGFR (CKDEPI, MDRD-4, CG) were lower in the group of patients who died compared to the group of survivors with a powerful statistical association with respect to mortality. The same results were shown in the sub analysis of the study Candesartan in Heart Failure: Assessment of Reduction in Mortality and Morbidity (CHARM), in which the risk of death, cardiovascular death and hospitalization due to heart failure increased independently and significantly as filtering decreased glomerular, both in patients with depressed and preserved systolic function [9].

The decreased diuretic rhythm appeared more frequently in deceased patients, this is related to the presence of shock and hypovolemia, which is one of the most frequent causes of acute renal dysfunction in therapies. The kidney requires a continuous plasma flow through it, not only to maintain adequate values of glomerular filtration, but to preserve the integrity of its parenchyma. Faced with small fluctuations in this organ, it sets in motion a series of mechanisms with a view to maintaining perfusion in vital organs of the economy, thus depriving itself of the perfusion necessary to maintain its function, thus favoring the presence of ARF and a worse prognosis [5].

In relation to the survival time in the ICU, patients who presented ARF died early with respect to those who did not, with a statistically difference between both groups of studies (Figure 1). On the other hand, in the Cox regression analysis, the decrease in eGFR (CK-EPI) was only statistically associated with a higher risk of dying in the ICU (AHR 0.92 [0.85-0.99], $\mathrm{p}=0.026$ ). We think that this rate is more precise and accurate, since the eGFR (CG) depends on the estimations of the weight or the ideal weight, being able to complicate the calculation and the presentation of the results. Regarding eGFR (MDRD4) both in its complete and abbreviated form, easily obtainable laboratory data are used, but systematically underestimate the glomerular filtration rate when serum creatinine concentrations are very high, which can lead to misdiagnosis [16].

The limitations of this work are those of observational studies that do not allow a stratified randomization, together with the fact that the study performed was restricted to only one ICU, however, we consider a population with diverse medical, surgical, traumatic and infectious conditions, which represent the most common causes of ARF in critically ill patients [17]. To our knowledge, this work is the first attempt to systematically examine the magnitude and implications of changes in eGFR as an indicator of acute renal damage associated with the prognosis of the critical patient. 
Citation: Gutiérrez HB, Navas JL (2018) Predictive Value of Renal Function and Glomerular Filtration Rates in the Intensive Care Unit. J Kidney 4: 170. doi:10.4172/2472-1220.1000170

Page 5 of 5

\section{Conclusion}

The assessment of renal function by determining the estimation of glomerular filtration rate with eGFR (CK-EPI) provides useful and very valuable information for the initial evaluation of the critical patient. Any decrease in eGFR (CK-EPI) reflects an increased probability of death in this type of patient. It is, therefore, a marker of immediate, easy and available in all health centers.

\section{References}

1. MasFont S, Ros-Martinez J, Perez-Calvo C, Villa-Diaz P, Aldunate-Calvo S, et al. (2017) Prevention of acute kidney injury in Intensive Care Units. Medicina Intensiva 41: 116-126.

2. Venegas Justiniano JY, Hurtado AA (2013) Clinical characteristics of patients with acute renal injury in the Intensive Care Unit. Rev Soc Peru Med Interna 26: 121-126.

3. Doyle JF, Forni LG (2016) Acute kidney injury: Short-term and long-term effects. Critical Care 20: 188.

4. Gehr TWB, Schoolwerth AC (2017) Clinical assessment of renal function. Textbook of Critical Care. Barcelona: Elsevier 7: 712-717.

5. Betancourt AN, Mazon LG (2015) Evaluation of acute renal failure according to the criteria "RIFLE" in patients admitted to intensive care. Cuban J Intensive Med Emerg 14: 70-85.

6. Lopez Brinas E (2012) Acute renal failure. Internal Medicine Barcelona: Elsevier: 809-816.

7. Palevsky PM (2014) Acute Kidney Injury. Principles of Diagnosis and Management in the Adult. Barcelona: Elsevier 965-971.
8. Perez Salazar GG, Morales Quintana CF, Garcia Estrada CF, Mejicanos Quinonez JA, Gutierrez S, et al. (2013) Acute renal failure: Characterization and predisposing factors in patients in the intensive care unit. Rev med Internal 17: 13-21.

9. Flores Blanco PJ (2016) Prognostic role of new methods for assessing renal function in acute heart failure and acute coronary syndrome without ST-segment elevation.

10. Seller Perez G, Herrera Gutierrez ME, Arias Verdu MD, De la Torre Prados MV, Cota Delgado (2017) Pathophysiology of acute renal failure. Treaty of Intensive Med Barcelona: Elsevier: 395-399.

11. Kidney Disease: Improving Global Outcomes (KDIGO) Acute Kidney Injury Work Group. KDIGO clinical practice guideline for acute kidney injury (2012) Kidney Int Suppl 2: 132.

12. Comignani P, Gambarte $G$ (2011) Renal insufficiency in situations related to the Coronary Unit Fascicle No. 2: 37-46.

13. Leon Ponce MA, Vega AN, Calderon JM, Santillan AA, Diaz JI, et al. (2008) Acute renal failure in trauma. Critical Med 22: 36-41.

14. Regueira T, Andresen M, Mercado M, Downey P (2011) Pathophysiology of acute renal failure during sepsis. Intensive Med 35: 424-432.

15. Bellomo R, Kellum JA, Ronco C, Wald R, Martensson J, et al. (2017) Acute kidney injury in sepsis. Intensive Care Med 43: 816-828.

16. Andry DW, Bazari H (2017) Approach to the patient with kidney disease. In: Goldman-Cecil Elsevier 114: 728-736.

17. Uchino S, Kellum JA, Bellomo R, Doig GS, Morimatsu H, et al. (2005) Beginning and Ending Supportive Therapy for the Kidney (BEST Kidney) Investigators: Acute renal failure in critically ill patients: A multinational, multicenter study. JAMA 294: 813-818 